\title{
IV Brazilian Meeting on Simulational Physics
}

\section{Foreword}

This special issue includes the Proceedings of the IV Brazilian Meeting on Simulational Physics, which was held in the city of Ouro Preto, Minas Gerais, Brazil, during the period 9 - 12 August, 2005. This was the fourth meeting in the series, and the second to have invited papers, as well as some selected contributions, published by the Brazilian Journal of Physics.

In addition to the previous meetings in the BMSP series (held in 1997 and 1999 in Belo Horizonte, and in Ouro Preto in 2003), Minas Gerais also hosted the International Conference on Computational Physics and Cellular Automata (Ouro Preto, 1989) and the Ising Centennial Colloquium celebrating the 100th year of the birth of Ernst Ising (Belo Horizonte, 2000). All of these meetings were well attended and scientifically valuable, and demonstrate the strength of the Brazilian physics community.

The fourth BMSP brought together more than 70 researchers from all over the world for a pleasant and productive period. The talks and posters highlighted recent advances in applications, algorithms, and implementations of computer simulation methods for the study of condensed matter materials and biological motivated systems. We are sure that this series will continue to represent an important venue for people working in simulational physics, to exchange ideas and discuss the state of art of this always expanding field.

We are again very glad to have this special issue ready and most grateful to Professor Silvio Salinas, the editor of the Brazilian Journal of Physics, for making this publication possible. We are most grateful to Professor Américo Tristão Bernardes and the Universidade Federal de Ouro Preto for kind hospitality, to FAPEMIG, UFMG and UFOP for financial support, and to all of the authors for their written contributions.

João Antonio Plascak

Ronald Dickman

Guest Editors 